\title{
TITLE:
}

\section{OCEANOGRAPHIC DATA AT THE S.M.B.L., 1971}

$\operatorname{AUTHOR}(\mathrm{S})$ :

\section{CITATION:}

OCEANOGRAPHIC DATA AT THE S.M.B.L., 1971. PUBLICATIONS OF THE SETO MARINE BIOLOGICAL LABORATORY 1974, 21(5-6): 433-439

\section{ISSUE DATE:}

1974-12-23

URL:

http://hdl.handle.net/2433/175870

RIGHT: 


\section{OCEANOGRAPHIC DATA AT THE S. M. B. L. , 1971}

Measurements of the surface sea water temperature were made every day at $9: 00$ in the morning at the fixed site on the south coast of the laboratory ground near the water intake of the laboratory by
Mr. Yoshirô Kashiyama,
Mr. Sôichi Moriyama,
Mr. Mitsuru Ohta,
Mr. Shôzô Sakai,
Mr. Hidetomo Tanase, and
Mr. Yoshikazu Yamamoto.

The salinity was measured by salinometer (Tsurumi CO., LTD. Type E2).

Meteorological observations were made similarly in the laboratory yard by Miss Junko Kondô.

The necessary correction after formal manuals of oceanographical and meteorological observations, as well as the typewriting of tables, were made by

Dr. Shin-ichirô Fuse.

Weather is shown in Beaufort notation, and wind force in Beaufort scale. The codes used are :

$\begin{array}{rll}\text { b Blue sky } & 0 \text { Calm } & \text { less than } 0.3 \mathrm{~m} / \mathrm{sec} \\ \text { bc Partly clouded } & 1 \text { Light air } & 0.3 \sim 1.5 \mathrm{~m} / \mathrm{sec} \\ \text { c Cloudy } & 2 \text { Light breeze } & 1.6 \sim 3.3 \mathrm{~m} / \mathrm{sec} \\ \text { o Overcast } & 3 \text { Gentle breeze } & 3.4 \sim 5.4 \mathrm{~m} / \mathrm{sec} \\ \text { d Drizzling rain } & 4 \text { Moderate breeze } & 5.5 \sim 7.9 \mathrm{~m} / \mathrm{sec} \\ \text { r Rain } & 5 \text { Fresh breeze } & 8.0 \sim 10.7 \mathrm{~m} / \mathrm{sec} \\ \text { s Snow } & & \\ \text { p Passing showers } & & \\ \text { q Squalls } & \end{array}$

Summarizing the data, the following annual values are obtained for 1971 :

\begin{tabular}{|c|c|c|c|}
\hline & Lowest & Highest & Mean \\
\hline Air temperature $\left({ }^{\circ} \mathrm{C}\right)$ & $4.0 \quad$ (Feb. & 31.2 (July 15) & 17.7 \\
\hline Water temperature $\left({ }^{\circ} \mathrm{C}\right)$ & $9.10($ Mar. 11) & 29.08 (Aug. 3) & 19.92 \\
\hline Salinity $(\%)$ & 29. 651 (May 19) & 35. 163 (Jan. 18) & 33.944 \\
\hline Total precipitation (min) & 1816.0 & & \\
\hline
\end{tabular}

Publ. Seto Mar. Biol. Lab., XXI, 433-439, 1974. (Oceanographic data) 


\section{JANUARY}

date weather air tem- precipi${ }^{\circ} \mathrm{C}$ tation $\mathrm{mm}$

perature ${ }^{\circ} \mathrm{C}$

$$
\mathrm{mm}
$$

$\begin{array}{lllll}8.8 & - & \text { NW } 1 & 16.50 & 34.668\end{array}$

$8.6 \quad$ NW $1 \quad 16.60 \quad 34.686$

$\begin{array}{llll}8.2 & -0 & 16.40 & 34.632\end{array}$

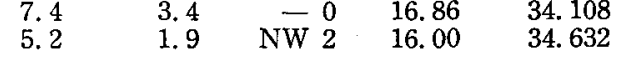

$\begin{array}{llllllll}5 & \mathrm{r} & & & & & & \\ 6 & \mathrm{bc} & 5.4 & 1.2 & \mathrm{~N} & 1 & 15.50 & 34.650 \\ 7 & \mathrm{c} & 6.1 & & & 0 & 15.24 & 34.632\end{array}$

$\begin{array}{rrrrrrr}6 & \text { bc } & 5.4 & 1.2 & \text { N } 1 & 15.50 & 34.650 \\ 7 & \text { c } & 6.1 & - & -0 & 15.24 & 34.632 \\ 8 & o & 7.8 & - & -0 & 14.90 & 34.596 \\ 9 & \text { b } & 7.1 & - & \text { NW } 1 & 14.16 & 34.596\end{array}$

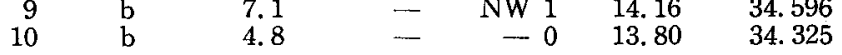

$\begin{array}{llrlrrr}11 & \mathrm{o} & 5.4 & - & \mathrm{NE} 1 & 15.42 & 34.469 \\ 12 & \mathrm{~b} & 8.5 & - & -0 & 15.76 & 34.747\end{array}$

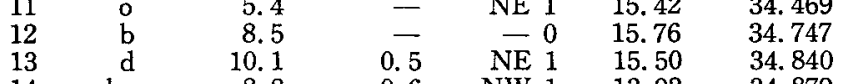

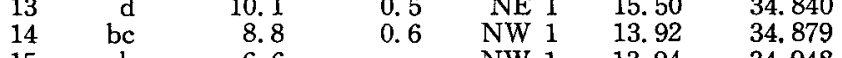

$\begin{array}{lllllll}15 & \mathrm{~b} & 6.6 & - & \text { NW } 1 & 13.94 & 34.948 \\ 16 & & 9.6 & - & \text { NW } & 13.98 & 35.013\end{array}$

$\begin{array}{rrrlrrr}16 & \mathrm{c} & 9.6 & - & \text { NW } 2 & 13.98 & 35.013 \\ 17 & \mathrm{bc} & 7.4 & - & \text { NW } 3 & 13.90 & 34.998 \\ 18 & \mathrm{~b} & 8.4 & - & \text { NW } 3 & 12.50 & 35.163\end{array}$

$\begin{array}{rrrrrrr}19 & \mathrm{c} & 10.9 & - & \text { NW } 2 & 13.36 & 34.934 \\ 20 & \mathrm{o} & 8.4 & - & -0 & 14.20 & 35.029\end{array}$

$\begin{array}{rrrrrrr}20 & \mathrm{o} & & -0 & 14.20 & 35.029 \\ & & & & & & \\ 21 & \mathrm{r} & 14.6 & 12.5 & -0 & 16.30 & 34.388\end{array}$

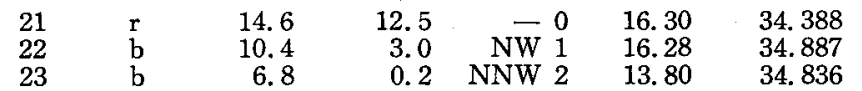

23
24
25

$\begin{array}{lllll}6.3 & - & \text { NW } 1 & 13.40 & 34.695 \\ 6.4 & - & \text { NE } 1 & 13.85 & 34.922\end{array}$

$\begin{array}{lr}26 & \text { b } \\ 27 & \text { bc } \\ 28 & \text { c } \\ 29 & \text { b } \\ 30 & \text { bc } \\ 31 & \text { bc }\end{array}$

mean $\begin{array}{llrll}6.4 & 3.2 & \text { NW } 2 & 13.30 & 34.870 \\ 6.4 & - & \text { N } 1 & 13.52 & 34.806 \\ 5.4 & - & \text { N } 2 & 11.80 & 34.851 \\ 5.2 & - & \text { N } 1 & 10.40 & 34.795 \\ 4.6 & - & \text { NW } 3 & 10.80 & 34.637 \\ 6.4 & - & \text { NW } 2 & 11.20 & 34.448\end{array}$

$\begin{array}{lll}7.5 & 14.29 & 34.732\end{array}$

\section{FEBRUARY}

date weather air tem- precipi- wind water tem- salinity perature tation perature

$\%$

$\begin{array}{lllll}4.7 & - & \text { NW } 2 & 11.27 & 34.590 \\ 4.8 & - & \text { NW } 1 & 13.90 & 34.875\end{array}$

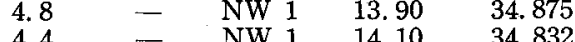

$\begin{array}{llll}4.4 & -\quad N W 1 & 14.10 & 34.832\end{array}$

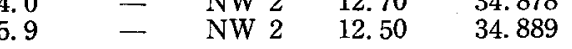

$6.5 \quad-\quad$ NW 1 1 $12.60 \quad 34.898$

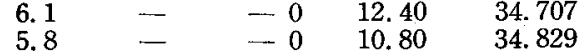

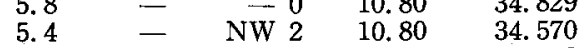

6.0 - NW 2 11.10 34.562

$\begin{array}{llll}5.3-N W 1 & 12.70 & 34.964\end{array}$

$\begin{array}{rrrrr}7.2 & - & -0 & 12.90 & 34.602 \\ 10.6 & 2.6 & \mathrm{NW} 2 & 13.60 & 34.573\end{array}$

$\begin{array}{lllll}8.6 & - & -0 & 14.58 & 34.611\end{array}$

$\begin{array}{lllll}12.8 & 12.0 & \text { NW } 1 & 14.90 & 34.443\end{array}$

$\begin{array}{lllll}8.9-0 & - & -13.40 & 34.561\end{array}$

$\begin{array}{rrrrr}14.2 & 0.7 & \mathrm{~N} 1 & 14.60 & 34.533\end{array}$

$\begin{array}{lllll}9.3 & 17.5 & \mathrm{~N} 2 & 13.68 & 34.732\end{array}$

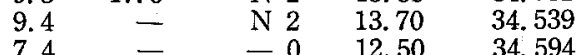

10.3 - E 1 1 $13.52 \quad 34.433$

$\begin{array}{lllll}12.7 & 9.1 & \text { E } 1 & 14.40 & 30.955\end{array}$

$\begin{array}{lllll}17.8 & 8.0 & \mathrm{~S} 2 & 16.20 & 34.607\end{array}$

$\begin{array}{lllll}10.2 & 5.2 & \mathrm{~N} 2 & 15.00 & 34.669\end{array}$

$\begin{array}{llll}-0 & -0 & 13.80 & 34.879\end{array}$

$\begin{array}{lllllll}26 & \mathrm{r} & 10.1 & 5.0 & -0 & 13.80 & 34.228 \\ 27 & \mathrm{~b} & 11.6 & 6.5 & \mathrm{~N} & 14.80 & 34.724\end{array}$

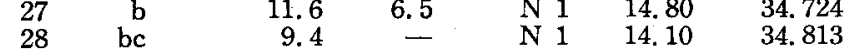

mean

8. 4

$13,37 \quad 34.540$ 


\section{MARCH}

$\begin{array}{llll}\text { date weather } & \begin{array}{l}\text { air tem- } \\ \text { perature }\end{array} & \begin{array}{l}\text { precipi- } \\ \text { tation }\end{array} & \begin{array}{c}\text { wind water tem- salinity } \\ \text { perature }\end{array}\end{array}$ perature ${ }^{\circ} \mathrm{C}{ }^{\text {tation }} \mathrm{mm}$

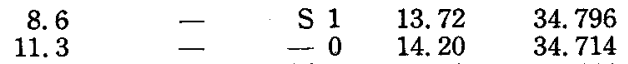$$
\begin{array}{lllll}
14.0 & 29.0 & \mathrm{~N} 2 & 17.10 & 34.444
\end{array}
$$$$
\begin{array}{lllll}
7.4 & - & \text { N } 3 & 12.40 & 34.650 \\
6.8 & - & \text { N } 2 & 12.36 & 34.642
\end{array}
$$

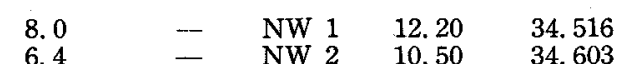

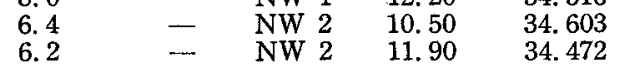$$
\begin{array}{llll}
9.0 & -\quad \mathrm{N} 2 & 10.00 & 34.248
\end{array}
$$$$
\begin{array}{rrrrr}
7.3 & - & N & \\
7 & & & 10.00 & 34.248 \\
\hline
\end{array}
$$$$
\begin{array}{rrrr}
6.1 & -\quad \mathrm{N} 1 & 9.10 & 34.243
\end{array}
$$$$
\begin{array}{lllll}
6.8 & - & 0 & 10.50 & 34.267
\end{array}
$$$$
\begin{array}{llll}
8.9 & 0.5 & \mathrm{~N} 2 & 11.70
\end{array}
$$$$
\begin{array}{lllll}
8.3 & - & & & \\
5.3 & & 10.70 & 34.423 \\
7.0 & - & N W 2 & 10.90 & 34.519
\end{array}
$$

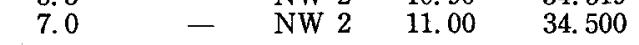$$
\begin{array}{lllll}
11.9 & & \text { NW } 1 & 12.24 & 34.484 \\
10.4 & 0.8 & \text { NW } 3 & 13.20 & 34.577
\end{array}
$$$$
\begin{array}{lll}
0.8-N W 3 & 13.20 & 34.577
\end{array}
$$

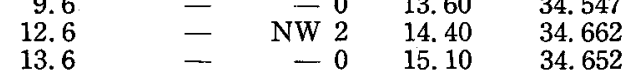

$\begin{array}{llrrr}15.1 & -0 & 14.92 & 34.692\end{array}$

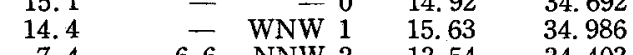
$\begin{array}{lllll}7.4 & 6.6 & \text { NNW } 2 & 13.54 & 34.403\end{array}$ $\begin{array}{rrrrr}7.8 & 1.7 & \text { NW } 2 & 12.42 & 34.403\end{array}$ $\begin{array}{llll}10.3 & - & \text { NW } 1 & 13.70\end{array}$

$\begin{array}{lllll}15.1 & & \text { SW } 1 & 15.80 & 34.681\end{array}$

$$
\begin{array}{lllll}
15.8 & 13.1 & \text { NW } 2 & 16.28 & 34.527
\end{array}
$$$$
\begin{array}{lllll}
15.4 & - & \text { SW } 1 & 15.51 & 34.564
\end{array}
$$$$
\begin{array}{llll}
19.4 & \text { SE } 2 & 16.94 & 34.609
\end{array}
$$$$
\begin{array}{lllll}
13.8 & 17.5 & \text { NW } 1 & 16.14 & 34.318
\end{array}
$$

10.6

13.32

34.512
APRIL

date weather air tem- precipi- wind water tem- salinity perature tation perature ${ }^{\circ} \mathrm{C} \quad \%$

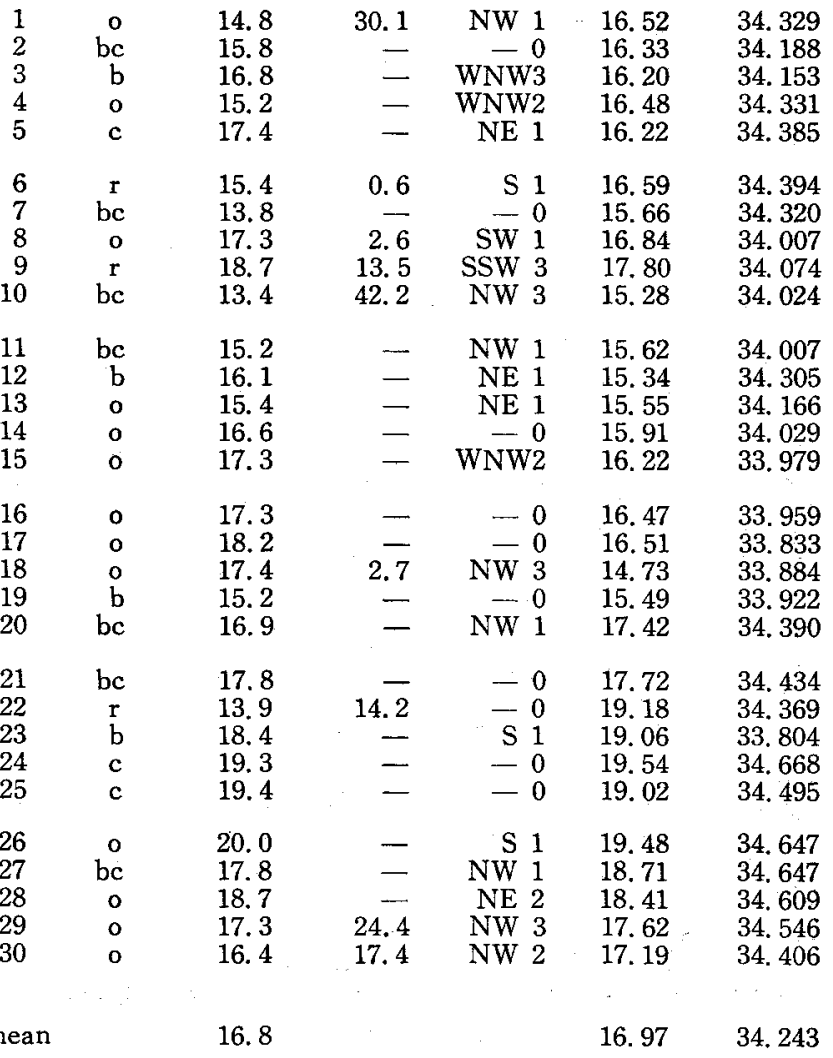


date weather air tem- precipi${ }^{\circ} \mathrm{C}$ perature tation

$\begin{array}{lrl}17.2 & - & \\ 19.9 & - & \\ 20.6 & - & \\ 19.9 & 2.2 & \\ 15.6 & - & \end{array}$

wind water temperature

salinity

NW $3 \quad 17.43$

SW 1 1 19.76

SSW 219.55

NW $2 \quad 17.33$

$15.9 \quad 1.8$

$\begin{array}{lr}16.6 & 10.9 \\ 18.7 & - \\ 15.2 & -\end{array}$

$\begin{array}{rrr}-0 & 18.29 \\ \text { NW } 3 & 18.09\end{array}$

$\begin{array}{ll}\text { NW } 3 & 18.09 \\ \text { NW } 3 & 18.50\end{array}$

NW $3 \quad 18.50$

$\begin{array}{ll}-0 & 17.77 \\ -0 & 18.72\end{array}$

21.7 -

21.8

$\begin{array}{rr}-0 & 19.62 \\ -0 & 20.72\end{array}$

S $1 \quad 20.69$

50.62
-0
-019.84

$\begin{array}{ll}22.3 & -\overline{0} \\ 16.6 & 4.0\end{array}$

19. $7 \quad 4.0$

22.

21. 2

$18.4 \quad 23.2$

20.8

25. 3

$\begin{array}{ll}21.6 & - \\ 22.1 & - \\ 18.4 & 1.8 \\ 20.4 & 6.1 \\ 22.1 & 8.4\end{array}$

23. $2 \quad-$

18.861 .2

$19.6 \quad 0.2$

$21.2 \quad 0.2$

19.7

34.452

34.263

34.416

34.221

34.463

34. 080

33.742
33.949

34.275
34.352

34. 377

34. 315

34. 363

34. 371

$\begin{array}{ll}0 & 19.50\end{array}$

19.50
-0

20.77

$\therefore \quad 21.54$

34. 367

34. 293

34.247

29.651

SSW $1 \quad 21.17 \quad 34.236$

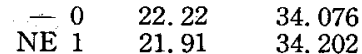

$\begin{array}{lll} & 21.91 & 34.202 \\ - & 21.41 & 34.315\end{array}$

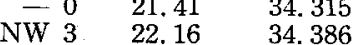

02208
-0

NE $1 \quad 21.78$

$\begin{array}{ll}-0 & 20.99 \\ -0 & 21.72\end{array}$

34.523

34.303

33.601

33. 468

33. 412

20.24

33.963
JUNE

date weather air tem- precipi- wind water tem- salinity perature tation

$\begin{array}{ll}0 & 22.12\end{array}$

21.89
-0

$$
{ }^{\circ} \mathrm{C} \quad \mathrm{mm}
$$

\begin{tabular}{|c|c|c|c|c|}
\hline 23. 4 & - & -0 & 22.54 & 33. 488 \\
\hline 25.3 & - & NW 1 & 22.39 & 33.542 \\
\hline 18.6 & 11.2 & NE 1 & 21.28 & 32.261 \\
\hline & 153 & 53 & 21.20 & 33.651 \\
\hline
\end{tabular}

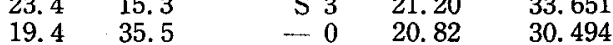

17.450 .2

$\begin{array}{ll}20.8 & 1.5 \\ 22.4 & - \\ 23.4 & 0.6\end{array}$

NW

20.21
-0

20.87
-0

$\begin{array}{rr} & \\ -0 & 22.07 \\ \mathrm{~S} 1 & 23.04\end{array}$

31. 233

32. 879

32.826

33.792
33.869

23. 3.2

$24.3 \quad 3.5$

$25.4 \quad-$

$22.8 \quad 0.3$

SW $2 \quad 23.83$

SW 1 22. 91

0
$-\mathrm{N}$

33.385

33. 877

33. 781 $20.4 \quad 16.5 \quad \mathrm{~N} 1$

$\begin{array}{lllll}22.4 & 54.0 & -0 & 22.23 & 32.758\end{array}$

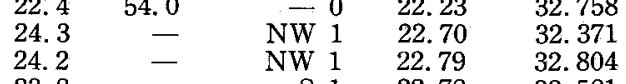

$\begin{array}{lll}\text { S } 1 & 22.78 & 33.581 \\ \text { E } 1 & 22.83 & 32.809\end{array}$

22.4 -

$\begin{array}{ll}22.9 & 2.3 \\ 22.6 & -\end{array}$

$\begin{array}{ll}22.6 & - \\ 22.6 & - \\ 22.9 & 1.0 \\ 23.3 & 6.4\end{array}$

NW $1 \quad 22.71$

33. 482

$\begin{array}{rrr}-0 & 22.90 \quad 32.836 \\ -0 & 23.06\end{array}$

$\begin{array}{lll}23.06 & 33.691 \\ - & 23.38\end{array}$

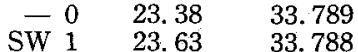

$25.8-$

33.869

26 bc

26

28
29

30

$24.7 \quad 0.5$

28. 8 -

$\begin{array}{rr}\mathrm{S} & 1 \\ \mathrm{NW} & 0\end{array}$

NW 24.22

SW 1.25 .02

33. 661

33.153

33. 783

mean

23. 1

22. 78

33. 197 
JULY $\begin{array}{llc}\text { date weather } & \begin{array}{l}\text { air tem- precipi- } \\ \text { perature tation }\end{array} & \text { wind water tem- salinity } \\ \text { perature }\end{array}$

perature ${ }^{\circ} \mathrm{C} \quad \%$

\begin{tabular}{|c|c|c|c|c|}
\hline $\begin{array}{l}24.8 \\
26.3 \\
28.4 \\
28.9 \\
28.8\end{array}$ & $\begin{array}{r}\overline{-} \\
\overline{-} \\
- \\
-\end{array}$ & $\begin{array}{rr}\mathrm{NE} & 1 \\
- & 0 \\
\overline{-} & 0 \\
\mathrm{NW} & 1 \\
\mathrm{~W} & 1\end{array}$ & $\begin{array}{l}24.14 \\
24.55 \\
24.80 \\
26.00 \\
26.10\end{array}$ & $\begin{array}{l}33.613 \\
33.597 \\
33.431 \\
33.328 \\
33.439\end{array}$ \\
\hline $\begin{array}{l}28.2 \\
26.8 \\
27.8 \\
27.5 \\
27.3\end{array}$ & $\begin{array}{r}28 . \overline{8} \\
10.6 \\
2.5 \\
-\end{array}$ & $\begin{aligned} & \text { S } 1 \\
& \text { ENE } 1 \\
& \text { S } 1 \\
& \text { S } 1 \\
& \text { S } 1\end{aligned}$ & $\begin{array}{l}26.64 \\
25.60 \\
25.62 \\
26.80 \\
25.86\end{array}$ & $\begin{array}{l}33.237 \\
32.469 \\
33.064 \\
33.236 \\
33.540\end{array}$ \\
\hline $\begin{array}{l}27.5 \\
27.8 \\
27.8 \\
30.1 \\
31.2\end{array}$ & $\begin{array}{l}- \\
E \\
-\end{array}$ & $\begin{array}{rr}\mathrm{S} & 0 \\
\mathrm{~W} & 1 \\
\mathrm{NW} & 1 \\
\mathrm{~W} & 1\end{array}$ & $\begin{array}{l}26.40 \\
26.10 \\
26.10 \\
26.42 \\
26.10\end{array}$ & $\begin{array}{l}33.466 \\
33.729 \\
33.682\end{array}$ \\
\hline $\begin{array}{l}29.4 \\
29.2 \\
29.5 \\
28.4 \\
26.4\end{array}$ & $\frac{-}{-}$ & $\begin{array}{rl}\text { NW } & 1 \\
\text { S } & 1 \\
\text { S } & 1 \\
\text { NW } & 1 \\
W & 2\end{array}$ & $\begin{array}{l}26.05 \\
25.00\end{array}$ & $\begin{array}{l}33.464 \\
33.888\end{array}$ \\
\hline $\begin{array}{l}24.6 \\
28.1 \\
24.8 \\
24.5 \\
26.4\end{array}$ & $\begin{array}{r}1.8 \\
-\overline{39.5} \\
96.2 \\
73.4\end{array}$ & $\begin{array}{c}\text { NW } 1 \\
\text { NE } 1 \\
\text { NE } 1 \\
\text { W } 1 \\
\text { S } 2\end{array}$ & $\begin{array}{l}23.50 \\
24.31 \\
25.45 \\
25.10 \\
24.75\end{array}$ & $\begin{array}{l}33.942 \\
33.670 \\
33.388 \\
30.114 \\
32.928\end{array}$ \\
\hline $\begin{array}{l}27.4 \\
27.8 \\
28.0 \\
28.8 \\
28.4 \\
29.3\end{array}$ & $\begin{array}{c}0.9 \\
- \\
- \\
-\end{array}$ & $\begin{array}{rl}\mathrm{S} & 1 \\
\mathrm{~S} & 0 \\
\mathrm{~S} & 1 \\
\mathrm{~S} & 1 \\
-\mathrm{S} & 0\end{array}$ & $\begin{array}{l}24.60 \\
25.42 \\
26.72 \\
27.79 \\
28.22 \\
28.31\end{array}$ & $\begin{array}{l}33.065 \\
33.106 \\
32.961 \\
33.269 \\
33.282 \\
33.195\end{array}$ \\
\hline 27.7 & & & 25.84 & 33.223 \\
\hline
\end{tabular}

AUGUST

date weather air tem- precipiperature tation

wind water tem- salinity $\mathrm{mm}$ perature ${ }^{\circ} \mathrm{C} \quad \%$

\begin{tabular}{|c|c|c|c|c|}
\hline 30.7 & - & $\mathrm{NE} 1$ & 28.42 & 33.471 \\
\hline 30.3 & & S 1 & 27.82 & 33. 263 \\
\hline 30.4 & 2.5 & ESE 1 & 29.08 & 33. 514 \\
\hline 31.0 & 0.5 & $\mathrm{NE} 2$ & 28.60 & 33. 399 \\
\hline 29.7 & 5.5 & S 3 & 28.30 & 33.621 \\
\hline 27.3 & 21.0 & S 3 & 27.46 & 31.958 \\
\hline 27.2 & 3.5 & S 1 & 27.60 & 33.522 \\
\hline 29.4 & - & -0 & 28.30 & 33.490 \\
\hline 28.3 & - & S 1 & 27.80 & 33.094 \\
\hline 29.4 & $\ldots$ & S 2 & 27.60 & 33.266 \\
\hline 28.4 & 0.8 & S 1 & 27.80 & 33.708 \\
\hline 28. 6 & 0.6 & S 1 & 28.10 & 33.600 \\
\hline 29.6 & - & N 1 & 27.90 & 33.703 \\
\hline 29.9 & - & $\mathrm{N} 1$ & 27.80 & 33. 723 \\
\hline 28.9 & - & -0 & 27.74 & 33.861 \\
\hline 30.5 & - & -0 & 27.94 & 33. 287 \\
\hline 26.8 & 0.5 & N 1 & 26.80 & 33.500 \\
\hline 27.8 & - & -0 & 26.28 & 33.404 \\
\hline 24.4 & 1.8 & NE 2 & 26.02 & 33.465 \\
\hline 24.6 & 2.7 & NE 2 & 25.53 & 33.608 \\
\hline 25.8 & - & -0 & 26.10 & 33. 699 \\
\hline 27.8 & - & -0 & 26.96 & 33.892 \\
\hline 28.6 & - & -0 & 27. 30 & 34.020 \\
\hline 28. 6 & - & -0 & 27.50 & 34.090 \\
\hline 27.8 & - & & 27.60 & 34.145 \\
\hline 25.8 & 4.0 & -0 & 26.63 & 34.001 \\
\hline 26.8 & 0.7 & -0 & 26. 70 & 34. 057 \\
\hline 29.7 & - & W 1 & 27.20 & 33. 791 \\
\hline 29.9 & - & N 1 & 27.56 & 33.795 \\
\hline 24.3 & 32.0 & W 3 & 25.50 & 32.500 \\
\hline 23.3 & 111.6 & N 4 & 25.70 & 31.807 \\
\hline 28. & & & 27.34 & 33. 492 \\
\hline
\end{tabular}


SEPTEMBER

date weather air tem- precipi- wind water tem- salinity perature tation

${ }^{\circ} \mathrm{C}$

$\begin{array}{ll}25.3 & 0 . \\ 24.6 & \\ 26.1 & \\ 25.8 & \\ 27.6 & 6.8\end{array}$

$\begin{array}{lll}-0 & 26.28 & 32.685\end{array}$

WSW $\begin{array}{lll}0 & 26.30 & 32.989\end{array}$

$\begin{array}{lllll}26.6 & 0.2 & \text { SSW } 2 & 26.10 & 33.579\end{array}$

$\begin{array}{lllll}24.8 & 0.9 & -0 & 25.93 & 33.213\end{array}$

$\begin{array}{lllll}24.8 & 0.9 & -0 & 25.93 & 33.213 \\ 25.4 & 2.4 & -0 & 26.14 & 33.231\end{array}$

$25.8-2.4 \quad 0 \quad 26.14-33.231$

$22.8 \quad 5.4 \quad$ ENE 3 25. 04

$22.8 \quad 5.4$

ENE 3

25.54

33. 238

$\begin{array}{rrrrr}25.5 & 12.2 & \overline{0} & 25.80 & 33.457 \\ 26.5 & 0.2 & \mathrm{NW} & 25.42 & 33.517\end{array}$

$\begin{array}{rrrrr}26.5 & 0.2 & \text { NW } 1 & 25.42 & 33.517 \\ 25.2 & - & \text { N } 2 & 25.30 & 33.025\end{array}$

$\begin{array}{lll}\text { N } 2 & 25.30 & 33.025 \\ -0 & 25.30 & 33.539\end{array}$

$\begin{array}{lll}-0 & 25.30 & 33.539 \\ -0 & 33.400\end{array}$

$22.4-$

24. $24 . \overrightarrow{3.6}$

21. 720.3

$-0 \quad 25.15$

NE $2 \quad 25,19$

NE $3 \quad 24.57$

- 025.55

32.938

33. 210

32.586

33.518

33. 601

\begin{tabular}{rrr}
-0 & 26.32 & 33.802 \\
\hline
\end{tabular}

$-0$

S 2 26.00 33.628

S $2-26.40$

$26.70 \quad 33.782$

$\begin{array}{llll}26 & \mathrm{r} & 22.7 & 18.0\end{array}$

$\begin{array}{rr}27 & c \\ 28 & b c \\ 29 & \text { o } \\ 30 & \text { b }\end{array}$

24. $7 \quad 147.0$

23.4 -

$22.6 \quad-$

mean

24.9

NW 2 25.50 24.67

$23.61 \quad 32.935$

NW $1 \quad 24.27 \quad 33.343$

25. $64 \quad 33.163$
OCTOBER

date weather air tem- precipi- wind water tem- salinity perature tation

perature

$\%$

$\begin{array}{lllll}17.4 & 3.0 & \text { WSW2 } & 23.82 & 32.734\end{array}$

$\begin{array}{lllll}20.4 & 22.2 & \text { NE } 2 & 24.37 & 33.255\end{array}$

$19.9-\mathrm{ENE} 1-23.50 \quad 33.362$

$17.7 \quad 26.2 \quad$ NNE 2 23.43

$\begin{array}{lllll}18.4 & 0.4 & 0 & 23.01 & 34.285\end{array}$

$\begin{array}{lllll}20.2 & 2.3 & \text { NW } 1 & 23.88 & 34.097\end{array}$

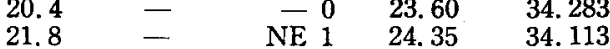

$\begin{array}{llrll}21.8 & - & & \\ 21.6 & 6.3 & -0 & 24.26 & 34.281\end{array}$

$\begin{array}{rrrrr}21.3 & 14.9 & -0 & 23.74 & 33.939\end{array}$

$\begin{array}{lllll}16.6 & 12.1 & \text { NW } 3 & 23.84 & 34.659\end{array}$

$\begin{array}{lllll}18.6 & - & \text { NW } 2 & 22.04 & 34.470 \\ 17.8 & 18.3 & \text { NW } 2 & 22.82 & 33.555\end{array}$

$\begin{array}{rrrrr}17.8 & 18.3 & -0 & 22.73 & 34.031\end{array}$

$\begin{array}{lllll}18.9- & -0 & 21.76 & 32.870\end{array}$

$19.6 \quad-\quad \mathrm{NW} 1$ 22.94

$19.1 \quad-$

$\begin{array}{lllll}19.1 & - & -0 & 22.12 & 33.204 \\ 18.9 & - & -0 & 21.66 & 33.475\end{array}$

$\begin{array}{lllll}19.4-N W 1 & - & 22.07 & 33.376\end{array}$

$\begin{array}{lllll}20.0 & & & & \\ 22.1 & 4.4 & & 21.76 & 33.458\end{array}$

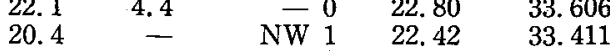

$\begin{array}{llll}18.4 \quad-\quad & -0 & 21.36 & 33.787\end{array}$

$\begin{array}{lllll}15.7 & 37.0 & \text { NNE } 2 & 21.40 & 33.449\end{array}$

$\begin{array}{lllll}16.4 & - & \text { NW } 2 & 20.85 & 33.146\end{array}$

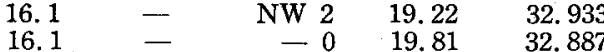

$\begin{array}{lllll}16.6 & -\overrightarrow{4} & \overrightarrow{\mathrm{NE}} 2 & 21.11 & 32.438\end{array}$

$\begin{array}{lllll}18.0 & 7.9 & \text { NE } 3 & 19.74 & 33.013\end{array}$

$\begin{array}{lll}18.9 & 22.49 & 33.531\end{array}$ 
NOVEMBER

date weather air tem- precipi- wind water tem- salinity perature tation

perature ${ }^{\circ} \mathrm{C} \quad \%$

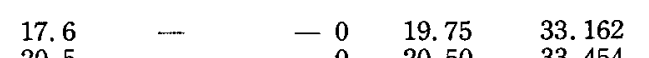

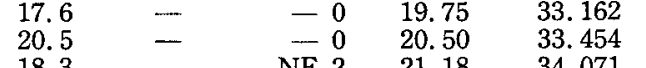

$\begin{array}{llll}18.3-N & \text { NE } 2 & 21.18 & 34.071\end{array}$

$\begin{array}{lllll}16.9 & 4.8 & \text { NE } 1 & 21.98 & 34.102 \\ 17.1 & - & \text { NW } 2 & 21.56 & 34.325\end{array}$

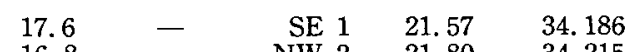

$\begin{array}{llll}16.8-N W 2 & 21.80 & 34.215\end{array}$

$\begin{array}{llll}14.6 & - & \text { NW } 3 & 18.52\end{array}$

$\begin{array}{llll}13.3 & \text { NW } 1 & 19.48 & 34.205\end{array}$

$\begin{array}{lllll}14.4 & 10.0 & -0 & 19.80 & 33.812 \\ 15.9 & - & -0 & 19.42 & 33.803\end{array}$

$\begin{array}{lllll}15.9 & - & -0 & 19.42 & 33.803 \\ 17.8 & - & -0 & 20.99 & 34.195\end{array}$

$\begin{array}{lllll}17.8 & - & -0 & 20.99 & 34.195 \\ 17.6 & 0.3 & -0 & 21.48 & 34.402\end{array}$

$\begin{array}{lllll}17.6 & 0.3 & -0 & 21.48 & 34.402 \\ 16.7 & 8.0 & \text { NW } 1 & 21.15 & 33.784\end{array}$

$\begin{array}{lllll}13.9 & 4.3 & \text { NW } 2 & 20.44 & 34.131\end{array}$

$\begin{array}{lllll}14.3-0 & -0 & 19.64 & 34.259\end{array}$

$\begin{array}{lllll}13.2 & 2.0 & \mathrm{~N} 1 & 19.51 & 34.343\end{array}$

$\begin{array}{lllll}14.8 & - & \text { NW } 2 & 19.22 & 34.161 \\ 13.8 & - & \text { NE } 1 & 19.85 & 34.062\end{array}$

$\begin{array}{llll}12.9 & \text { E } 1 & 19.72 & 34.103\end{array}$

$\begin{array}{llll}12.9 & \text { E } 1 & 19.72 & 34.103 \\ 15.7 & - & \text { NNW } 4 & 18.63\end{array}$

$12.8 \div \quad \mathrm{NNW} 418.63$

$\begin{array}{llll}12.8 & - & & \\ 14.3 & & 19.12 & 33.894 \\ 15.5 & 19.10 & 33.814\end{array}$

$\begin{array}{lllll}14.3 & - & \mathrm{NW} & 19.10 & 33.814 \\ 15.5 & - & \mathrm{NW} & 17.71 & 34.248\end{array}$

$\begin{array}{lllll}14.7 & - & \text { NW } 2 & 17.85 & 33.852\end{array}$

$\begin{array}{lllll}14.9 & - & \text { NE } 1 & 18.33 & 33.646 \\ 15.7 & - & \text { NW } 1 & 18.93 & 33.671\end{array}$

$\begin{array}{lllll}15.7 & - & \text { NW } 1 & 18.93 & 33.671\end{array}$

$\begin{array}{rrrrr}13.8 & - & \text { NW } 3 & 18.53 & 34.126 \\ 8.8 & - & \text { WNW } 4 & 15.49 & 34.250\end{array}$

15.2

19.69

34.003
DECEMBER

date weather air tem- precipi- wind water tem- salinity perature tation

perature ${ }^{\circ} \mathrm{C} \quad \%$

\begin{tabular}{|c|c|c|c|c|}
\hline $\begin{array}{l}11.3 \\
10.4 \\
15.9 \\
10.8 \\
11.3\end{array}$ & $\begin{array}{l}- \\
3.5 \\
-\end{array}$ & $\begin{array}{rr}\text { WNW } & 3 \\
\text { N } & 1 \\
\text { WNW } & 2 \\
N W & 2 \\
\text { NE } & 1\end{array}$ & $\begin{array}{l}15.75 \\
17.80 \\
18.81 \\
17.80 \\
18.14\end{array}$ & $\begin{array}{l}34.653 \\
34.691 \\
34.930 \\
34.682 \\
34.745\end{array}$ \\
\hline $\begin{array}{r}13.2 \\
7.4 \\
9.4 \\
8.8 \\
9.1\end{array}$ & $\begin{array}{l}0.4 \\
0.5 \\
- \\
- \\
\end{array}$ & $\begin{array}{rr} & 0 \\
\mathrm{~N} & 2 \\
\mathrm{~N} & 2 \\
\mathrm{~N} & 2 \\
\mathrm{NW} & 1\end{array}$ & $\begin{array}{l}17.90 \\
15.50 \\
17.00 \\
16.60 \\
16.51\end{array}$ & $\begin{array}{l}34.789 \\
34.656 \\
34.533 \\
34.668 \\
34.990\end{array}$ \\
\hline $\begin{array}{r}10.7 \\
12.0 \\
8.4 \\
9.8 \\
9.6\end{array}$ & $\begin{array}{r}54.0 \\
\overline{-} \\
1.7\end{array}$ & $\begin{array}{rr}\bar{W} & 0 \\
\mathrm{NW} & 1 \\
\overline{\mathrm{N}} & 0\end{array}$ & $\begin{array}{l}17.10 \\
16.82 \\
16.10 \\
16.80 \\
16.00\end{array}$ & $\begin{array}{l}34.965 \\
34.688 \\
34.998 \\
34.929 \\
34.923\end{array}$ \\
\hline $\begin{array}{r}8.4 \\
11.2 \\
11.8 \\
15.1 \\
11.8\end{array}$ & $\begin{array}{l}- \\
3.9 \\
1.6 \\
2.6\end{array}$ & 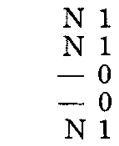 & $\begin{array}{l}15.50 \\
16.20 \\
17.30 \\
19.50 \\
17.30\end{array}$ & $\begin{array}{l}34.950 \\
35.035 \\
34.961 \\
34.946 \\
34.946\end{array}$ \\
\hline $\begin{array}{r}11.6 \\
8.8 \\
8.6 \\
7.9 \\
11.9\end{array}$ & $\begin{array}{l}- \\
z \\
-\end{array}$ & $\begin{array}{ll}N & 1 \\
N & 1 \\
N & 1 \\
- & 0 \\
- & 0\end{array}$ & $\begin{array}{l}17.20 \\
15.30 \\
15.30 \\
16.30 \\
16.30\end{array}$ & $\begin{array}{l}34.942 \\
35.044 \\
34.963 \\
34.962 \\
34.858\end{array}$ \\
\hline $\begin{array}{r}12.5 \\
12.4 \\
9.6 \\
4.2 \\
12.0 \\
12.4\end{array}$ & $\begin{array}{r}39.0 \\
6.2 \\
30.6 \\
1.5 \\
25.4\end{array}$ & $\begin{array}{rr}\mathrm{N} & 1 \\
-\mathrm{N} & 0 \\
- & 0 \\
- & 0 \\
\mathrm{NW} & 1\end{array}$ & $\begin{array}{l}17.40 \\
17.80 \\
16.50 \\
14.12 \\
16.88 \\
17.42\end{array}$ & $\begin{array}{l}32.803 \\
34.244 \\
34.553 \\
34.063 \\
34.518 \\
34.653\end{array}$ \\
\hline 10.6 & & & 16.80 & 34. 719 \\
\hline
\end{tabular}

